\title{
Comparative study of Cronobacter identification according to phenotyping methods
}

\author{
Emily E. Jackson and Stephen J. Forsythe
}

\begin{abstract}
Background: Microbiological criteria applied to powdered infant formula (PIF) require the absence of all Cronobacter spp. Consequently, misidentification of isolates from finished products can lead to significant financial losses for manufacturers and could increase the risk of neonatal infection. Biochemical identification of suspect isolates using commercially available test panels is recommended for use by PIF manufacturers by both the US FDA and ISO standard methods for Cronobacter species; however, phenotyping can be unreliable, particularly for a genus such as Cronobacter where the taxonomy has been subject to frequent changes. This study compared the predicted identification by commonly used phenotyping kits (API20E and ID32E) for over 240 strains of Cronobacter from diverse sources, which had been identified using DNA sequence analysis. In 2015, the databases associated with the API20E and ID32E biochemical test panels were updated, including the recognition of the Cronobacter genus. Thus, the identifications from multiple versions the databases were compared to each other and to identifications based on DNA sequencing methods.

Results: Using previous versions of the API20E database, $90.0 \%$ of strains (216/240) resulted in a match for the species identification; however, version 5.0 produced matches for only $82.3 \%$ of strains (237/288). Similarly, the update to version 4.0 in the ID32E database caused the percentage of matches to drop from $88.9 \%$ (240/270) to $43.2 \%$ (139/ 322). A smaller study showed that the Vitek GN system identified all 14 strains, belonging all seven Cronobacter species, as members of the 'C. sakazakii group,' but also attributed three strains of Franconibacter helveticus and F. pulveris to this group. In silco analysis of a PCR-based method targeting ompA predicted that amplification would only occur with Cronobacter species and this method may be a feasible alternative to biochemical phenotyping.

Conclusions: These results indicate that commercially available biochemical test panels are not sufficiently reliable for speciation of Cronobacter isolates. Although DNA-sequence based methods would be the more reliable approach; however, this is not currently feasible for many food microbiology laboratories. Instead, a previously published PCR-based method targeting ompA is suggested as an alternative for identification of Cronobacter species based on in silico analysis.
\end{abstract}

Keywords: Cronobacter, Phenotyping, Biochemical identification

\footnotetext{
* Correspondence: Stephen.forsythe@ntu.ac.uk

Pathogen Research Group, School of Science and Technology, Nottingham

Trent University, Clifton Lane, Nottingham NG11 8NS, UK
}

(c) 2016 The Author(s). Open Access This article is distributed under the terms of the Creative Commons Attribution 4.0 International License (http://creativecommons.org/licenses/by/4.0/), which permits unrestricted use, distribution, and reproduction in any medium, provided you give appropriate credit to the original author(s) and the source, provide a link to the Creative Commons license, and indicate if changes were made. The Creative Commons Public Domain Dedication waiver (http://creativecommons.org/publicdomain/zero/1.0/) applies to the data made available in this article, unless otherwise stated. 


\section{Background}

Current microbiological criteria applied to powdered infant formula (PIF) require the absence of all seven Cronobacter species in thirty $10 \mathrm{~g}$ samples [1]. Subsequently, the misidentification of microorganisms in PIF can lead to significant losses for manufacturers and may present a risk to neonates. The in-house false positive misidentification of an isolate as Cronobacter in a batch of product would result in the manufacturer losing productivity and profits. Whereas, a false negative identification, in which a Cronobacter isolate is misidentified as a permitted organism, may result in neonatal infections, product recalls, and lost consumer confidence. These losses can be significant for manufacturers as demonstrated in 2011 when a suspected outbreak of $C$. sakazakii in the United States led to product recalls and a subsequent $10 \%$ drop in the manufacturers' shares [2,3]. This was despite the lack of laboratory evidence to linking their product to infant infections and deaths $[2,3]$. The costs of infection are also significant, due to the long-term effects of the illness, including life-long brain damage. Minor et al. (2015) estimated the cost of C. sakazakii infections to be greater than $\$ 5$ million per case [4].

The Cronobacter genus is currently recognized as containing 7 species, whereas prior to 2007 all species within the genus were known as 'Enterobacter sakazakii'. The 2007 and 2008 taxonomic descriptions of the Cronobacter genus and its members used biotyping to re-assign Enterobacter sakazakii strains to the new species; however, biotyping has been reported to contradict DNA sequencebased phylogeny based on multilocus sequence typing and whole genome sequence analysis [5-8]. While DNA sequence-based methods are considered to be more reliable for identification of Cronobacter species, they are also more expensive, more labor intensive, and have a slow turnaround time. Consequently, it is not currently feasible for PIF manufacturers to employ these methods.

Biochemical identification of suspect Cronobacter isolates from PIF is often recommended. Previously, the 2006 ISO standard recommended use of the ID32E biochemical test panel, but the proposed new ISO standard specifies traditional microbiological methods for confirmation with seven required biochemical tests $[9,10]$. Six of these tests are included in the ID32E test panel and the proposed standard states that such kits can be used in place of more traditional biochemical methods [10]. Additionally, though the FDA Bacteriological Analytical Manual (FDA BAM) includes a real-time PCR screening method, the results must be confirmed culturally [11]. The recommended cultural methods can also be used independently for identification of suspect isolates when PCR-based methods are not available [11]. According to the FDA BAM, biochemical identification of suspect Cronobacter isolates should be carried out using the ID32E test kit or Vitek 2 GN cards [11].
Though they are widely used and recommended by both ISO and the FDA, biochemical test panels, like the ID32E, can be unreliable for various reasons. First, their reliance on visual detection of color changes make accurate identification difficult due to subjectivity when reading the test results. Additionally, discrepancies between the different test kits have also been reported. For example, Iversen et al. correctly identified $90 \%$ of Cronobacter isolates to the nearest match of 'Enterobacter sakazakii' with the ID32E test kit, but only $70 \%$ of those isolates were correctly identified with the API20E [12]. Finally, the accuracy of identification databases can be problematic due to changes in bacterial taxonomy which are not reflected promptly. This is a particular problem for the genera Cronobacter where a number of taxonomic changes have occurred very quickly and some species are very closely related [13-15]. Both the API20E and ID32E databases persisted in using the pre-2007 designation of ' $E$. sakazakii' until 2015. Following the 2015 update, version 5.0 of the API20E database reports a result of 'Cronobacter spp.' while version 4.0 of the ID32E database purports to identify isolates to the species level within the Cronobacter genus.

Despite the reliance on phenotyping methods for identification of Cronobacter spp. in PIF, no large scale analysis of the accuracy of such methods has been undertaken. The current study was conducted to compare the species identifications using both old and new versions of the API20E and ID32E databases. The biochemical profiles from over 240 previously identified strains in the culture collection at Nottingham Trent University (NTU) were analysed with the updated databases and the results were compared to the previous identifications. Additionally, a subset of 19 strains were analysed with Vitek GN cards and the results from all three biochemical methods were compared. A PCR probe-based method targeting the gene ompA was explored as an alternative method because it requires only a PCR thermocycler and gel electrophoresis capabilities, which are available in most microbiology laboratories. This method was analyzed in silico using 223 full genome sequences belonging to Cronobacter and related genera.

\section{Methods}

\section{Biochemical identification}

API20E and ID32E profiles and identifications were obtained from the archived records of the NTU culture collection. These profiles were re-analysed using version 5.0 of the API20E database and version 4.0 of the ID32E database. It was not feasible to determine all predicted identifications for recently acquired strains since the older database versions were no longer accessible. This resulted in differences in the number of total strains for some analyses. All strains used in this study have been 
identified to the species level using $16 \mathrm{~S}$ rDNA sequencing or sequencing of the fus $A$ allele as part of the multilocus sequence typing (MLST) scheme for Cronobacter spp. $[16,17]$. The strains used in these analyses were isolated from 21 different countries over a period of 65 years (1950-2015). These isolates were obtained from a variety of sources including PIF and PIF manufacturing environments, foods, herbs and spices, and clinical samples.

Due to the use of different versions of the associated databases, the date of the original analysis influences the species identification. For the older versions of both databases, a result of "Enterobacter sakazakii" was considered to be a match for all Cronobacter species and strains, as this was the closest identification available in the database at the time. A result of "Cronobacter spp." was considered as to be a match for all Cronobacter strains with the updated version of the API20E database; however, with the updated ID32E database, a result was only considered to be a match if the identified species matched the species as determined by one or more DNA sequence-based methods. Strains giving a result of "Unacceptable profile" were considered to be mismatches. Identifications with the older versions of the databases were not available for all strains. These results were assigned as "unknown" and were not considered to be either matches or mismatches. The $X^{2}$ test was used to determine if the percentage of matches differed significantly between database versions. Test statistics and $p$-values were calculated using Microsoft Excel. A $p$ value of $<0.05$ was considered to be significant. Only strains which had been designated as matches or mismatches were included in this analysis.

A selection of 19 strains, including 14 Cronobacter strains, were additionally analysed with the semi-automated Vitek system; Table 1. Strains were selected for this analysis because their biochemical profiles and corresponding identifications have been published previously [18-20]. The non-Cronobacter strains were included in the analysis as they have been previously misidentified as members of the Cronobacter genus [20]. The Vitek $\mathrm{GN}$ analyses were carried out according to the manufacturer's instructions using the Vitek 2 Compact machine and GN cards. Species identification was performed using version 05.04 of the Vitek software and version 05.00.011 of the Vitek GN database.

\section{In silico analysis of the ompA PCR method}

The BLAST function of the PubMLST Cronobacter database was used to extract the $о m p A$ sequences with 500 nucleotide flanking regions from 187 Cronobacter strains representing all seven species $[17,21]$. Additionally, the corresponding gene sequences were extracted from 36 strains of closely related organisms, including members of the Enterobacter, Citrobacter, Franconibacter, Siccibacter, and Yersinia genera. The GenBank accession number for

Table 1 Bacterial species and strains analysed with the Vitek GN system

\begin{tabular}{|c|c|c|c|c|}
\hline Species & Strain & Source & Country of isolation & Year \\
\hline Cronobacter condimenti & LMG26250' & Food & Slovakia & 2010 \\
\hline Cronobacter dublinensis & LMG $23823^{\top}$ & Environmental & Ireland & 2004 \\
\hline Cronobacter malonaticus & 8 & Weaning food & Czech Republic & 2004 \\
\hline Cronobacter muytjensii & 16 & Spice & Unknown & 2005 \\
\hline Cronobacter sakazakii & 4 & Clinical & Canada & 1990 \\
\hline Cronobacter sakazakii & 5 & Clinical & Canada & 1990 \\
\hline Cronobacter sakazakii & 12 & Clinical & Czech Republic & 2003 \\
\hline Cronobacter sakazakii & 1436 & Food & Turkey & 2010 \\
\hline Cronobacter sakazakii & 1437 & Food & Turkey & 2010 \\
\hline Cronobacter sakazakii & 1438 & Food & Turkey & 2010 \\
\hline Cronobacter turicensis & 9 & Weaning food & U.K. & 2003 \\
\hline Cronobacter turicensis & LMG23827 & Clinical & Switzerland & 2005 \\
\hline Cronobacter universalis & NCTC9529 & Water & U.K. & 1956 \\
\hline Cronobacter universalis & 1435 & Food & Turkey & 2010 \\
\hline Escherichia hermanii & 162 & Rice & U.K. & 2004 \\
\hline Franconibacter helveticus & $\operatorname{LMG}^{2} 3732^{\top}$ & Fruit powder & Switzerland & 2007 \\
\hline Franconibacter pulveris & 1393 & Ingredients & U.K. & 2011 \\
\hline Franconibacter pulveris & LMG24057 & Fruit powder & Switzerland & 2008 \\
\hline Siccibacter colletis & $\mathrm{NCTC} 14934^{\top}$ & Food ingredient & U.K. & 2011 \\
\hline
\end{tabular}


the ompA sequence used in the BLAST search is DQ000206 [22].

Extracted sequences were aligned in MEGA 6 and were examined for the presence of primer binding sites for the PCR primers ESSR-F and ESSR-R from the method described by Mohan-Nair and Venkitanarayanan $[22,23]$.

\section{Results and discussion}

Since previous version(s) of the API20E database identified strains as "Enterobacter sakazakii," this result was considered to be a match for all Cronobacter species. A significant difference $\left(p<0.05, X^{2}\right.$ test) was found in the percentage of strains producing a profile that resulted in a match with the different versions of the database. Only $82.3 \%$ of strains resulted in a match with version 5.0, while $90.0 \%$ of strains resulted in a match with previous version(s). These results are summarized in Additional file 1: Table S1. The majority of strains $(68.8 \%)$ produced matches with both the old and new versions of the database; however, $6.3 \%$ of strains produced profiles which resulted in match previously but a mismatch following the update to the API20E database. Only one strain $(0.4 \%)$ showed a mismatch in the archived records, and a match following the update to the database.

Strains of Cronobacter were misidentified as Enterobacter aerogenes, E. amnigenus, and E. cloacae with the previous version of the API20E database and as E. aerogenes, E. amnigenus, E. cancerogenus, E. cloacae, and Serratia liquefaciens with the updated version. Strains of E. cloacae and E. hormaechei were misidentified as Cronobacter spp. (or 'E. sakazakii') with both versions of the database. Though most profiles returned a percent identification for each species, seven profiles did not do so with the updated database, returning a result of "Unacceptable profile". These results were considered to be mismatches and such results incorporate further uncertainty into identification of isolates producing these profiles.

A total of 61 API20E profiles were observed for confirmed strains of Cronobacter species. All of these profiles were positive for amygdalin fermentation and negative for hydrogen sulfide production. More than $90 \%$ of these profiles were also positive for $\beta$-galactosidase (96.7 \%), arginine dihydrolase $(93.4 \%)$, citrate utilization (93.4\%), and D-glucose (95.1\%), D-mannitol (91.8\%), L-rhamnose (91.8\%), D-saccharose (93.4\%), D-melibiose (96.7\%), and L-arabinose $(93.4 \%)$ fermentation. Additionally, more than $90 \%$ of these profiles were negative for urease activity $(95.1 \%)$. The observed reactions for arginine dihydrolase, citrate utilization, hydrogen sulfide production, urease activity, and acid production from glucose, Dmannitol, L-rhamnose, D-saccharose, D-melibiose, and $\mathrm{L}$-arabinose match the original description of the Cronobacter genus [5, 6].
Of the 61 API20E profiles, the most common were 3305373, 3307173, and 3305173 which represented 60, 41 and 40 strains, respectively. Together, these three profiles were observed for more than half of the strains analysed (141/260; $54.2 \%)$. These profiles differed from one another by only two traits; gelatinase activity and inositol fermentation. Each of these profiles resulted in a species identification of " $E$. sakazakii" with version 4.0 of the database and were identified as Cronobacter spp. with version 5.0. Profile 3307173 was only observed for confirmed strains of Cronobacter, belonging to four different species; $C$. sakazakii $(n=34)$, C. malonaticus $(n=4), C$. turicensis $(n=2)$, and C. dublinensis $(n=1)$. In addition to $C$. sakazakii $(n=32)$ and $C$. malonaticus $(n=8)$, strains of E. hormaechei $(n=3)$ and E. cloacae $(n=1)$ were identified with profile 3305173 . Strains of C. sakazakii $(n=49)$, C. malonaticus $(n=7)$, C. turicensis $(n=4)$ and $E$. hormaechei $(n=6)$ produced profile 3305373. Therefore, while these profiles were frequently observed for Cronobacter spp., they are not necessarily specific to members of this genus and may be produced by members of the Enterobacter genus.

Though easily distinguished by colony morphology on Druggan-Iversen-Forsythe agar, Enterobacter isolates have been misidentified as members of the Cronobacter genus in the past. For example, two illnesses in Mexico were attributed to $C$. sakazakii using cultural and molecular identification methods [24]. Subsequent DNA sequence-based analysis of the patient isolates, however, identified them as members of the Enterobacter genus [25]. Similarly, Townsend et al. (2008), used 16S rDNA sequencing to identify 10 clinical strains of Enterobacter hormaechei that had been previously identified as $C$. sakazakii by phenotyping [26]. The correct identification and genotyping of these isolates revealed that a previously unrecognised noscomial outbreak of E. hormaechei had occurred [26]. These investigations show that clinical Enterobacter isolates have been mistaken for $C$. sakazakii and though infectious, these organisms are not addressed in the microbiological criteria applied to PIF [1, 25-29]. Although false positive results were observed for E. cloacae and E. hormaechei, these organisms have been isolated from infant formula and can result in neonatal infections $[25,26]$. Even though the the risk from these organisms in PIF has not been closely examined, they may present a hazard and their misidentification as Cronobacter spp. could result in rejection of an unsafe batch of formula.

For the ID32E biochemical kit, Fanjat et al. noted that variation in the $\mathrm{L}$-arabinose and $\alpha$-maltosidase tests resulted in misidentification of some Cronobacter isolates (then known as "E. sakazakii") when using version 2.0 of the ID32E database [30]. The update to version 3.0 reflected this variability, resulting in $100 \%$ correct 
identification of the isolates [30]. Conversely, updating the database to version 4.0 resulted in higher numbers of misidentifications of Cronobacter species $\left(p<0.05, X^{2}\right.$ test). With the previous versions of the ID32E database, $88.9 \%$ of strains produced a match (as E. sakazakii); however, this percentage drops to only $43.2 \%$ when the updated version of the database is used (Additional file 2: Table S2).

Over one third of strains (37.9\%) produced a match both in the culture collection records and with version 4.0 of the ID32E database; however, $36.6 \%$ of strains produced a match in the culture collection records, but a mismatch after the database was updated. As with the API20E results, only one strain $(0.3 \%)$ showed a mismatch in the archived culture collection records, but a match with the updated database.

The major limitation with the update to the ID32E database is that it attempts to identify strains to the species level, but members of the Cronobacter genus can be difficult to differentiate without highly specific methods. In particular, C. sakazakii and C. malonaticus are so closely related that they cannot be reliably differentiated using $16 \mathrm{~S}$ rDNA sequencing methods [16]. The ID32E biochemical test panel is simply not specific enough to differentiate the seven species of Cronobacter. If these results are examined to only the genus level, $82.3 \%$ of confirmed Cronobacter strains were assigned to the Cronobacter genus using version 4.0 of the ID32E database. Though this still represents a significant difference $(p<$ $0.05, X^{2}$ test) in the accuracy of identification from the archived culture collection records, it is considerably more accurate than identification to the species level.

With the ID32E test kit, Cronobacter strains were misidentified as Enterobacter cancerogenus, E. cloacae, and Stenotrophomonas maltophilia with the old version(s) of the database and as Buttiauxella agrestis, Citrobacter freundii, Cit. koseri, E. aerogenes, E. cancerogenus, E. cloacae, Escherichia vulneris, Leclercia adecarboxylata, Serratia liquefaciens, S. rubidea, and Stenotrophomonas maltophilia with the updated database. Meanwhile, strains of Cit. freundii, Cit. koseri, E. hormaechei, Escherichia hermanii, Franconibacter helveticus, F. pulveris, Leclercia adecarboxylata, Pantoea spp., Siccibacter colletis, and Sic. turicensis were misidentified as Cronobacter species with the previous version of the database while Cit. freundii, Cit. koseri, E. hormaechei, E. pyrinus, Esh. hermanii, F. helveticus, L. adecarboxylata, and Siccibacter turicensis were misidentified as Cronobacter species with the updated version of the database. Misidentification of Enterobacter strains as Cronobacter spp. also occurred with the API20E test kit and the implications of such misidentifications were discussed previously. The misidentifications of Franconibacter and Siccibacter species as members of the Cronobacter genus is unsurprising as these species were briefly considered to be part of the Cronobacter genus [13-15, 20]. Fortyeight ID32E profiles returned a species identification with a description of "Unacceptable profile" instead of a percent identification with the updated version of the database. As with the API20E profiles, this produces more uncertainty in the species identification resulting from those profiles.

A total of 155 ID32E profiles were observed for confirmed strains of Cronobacter species. Nearly all (152/ $155,98.1 \%$ ) of the profiles give a positive result for $\beta$ glucuronidase activity. Similarly, only one profile $(0.7 \%)$ has a positive reaction for rhamnose acidification. More than $90 \%$ of the profiles for known Cronobacter spp. produced positive results for ornithine decarboxylase (90.3\%), arginine dihydrolase (94.2\%), $\beta$-glucosidase (98.1\%), malonate utilization (95.5\%), and acidification of maltose (91.0\%), D-glucose (91.0\%), D-saccharose (97.2 \%), L-arabinose (93.6\%) and D-arabitol (90.3\%). Additionally, more than $90 \%$ of these profiles were negative of L-aspartic acid arylamidase (97.4 \%), $\alpha$ galactosidase $(92.3 \%)$, and acidification of both Larabitol (97.4\%), and 5-ketogluconate (94.3\%). The observed reactions for ornithine decarboxyase, arginine hydrolase, maltose utilization and acidification of $\mathrm{D}$-glucose and L-arabinose match the original genus description of Cronobacter; however, the results for the acidification of L-rhamnose and D-arabitol do not match the original description of the genus $[5,6]$. These differences may be due to the use of different phenotyping methods when defining the genus. Additionally, the results for 4-nitrophenyl- $\alpha$-D-glucopyranoside, and acidification of D-arabitol and D-sorbitol differ significantly from the expected phenotypes specified in the proposed ISO standard [10]. While only $58.7 \%$ of Cronobacter ID32E profiles were positive for 4-nitrophenyl- $\alpha$-D-glucopyranoside, the ISO standard states that $100 \%$ of strains produced a positive result for this test [10]. Similarly, $74.2 \%$ of ID32E profiles were found to be positive for the acidification of $\mathrm{D}$ sorbitol; however, none of the strains included in the ISO standard produced a positive result [10]. Finally, nearly all of the profiles $(90.3 \%)$ were positive for the acidification of $\mathrm{D}$-arabitol, but only a few strains of $C$. dublinensis, C. turicensis, and C. universalis were reported to produce positive results in the ISO standard [10]. The presence of such discrepancies suggests that the biochemical test panel may not be completely accurate in its characterization of isolates. Importantly, biochemical test panels are suggested as an alternative to more traditional biochemical test methods in the draft ISO standard; however, the differences between the expected results given in the standard and the results reported here suggest that the ID32E test kit is 
not an acceptable alternative method for biochemical identification of suspect Cronobacter isolates [10].

The most common ID32E profile was 34276767250 $(n=34)$. Only C. sakazakii strains produced this profile, indicating a match to the species level. In addition to the tests matching the genus description given above, this profile is also positive for palatinose acidification, a characteristic given in the original description of $C$. sakazakii [6]. The remaining characteristics used for the description of the species are not included in the ID32E test kit. The next two most common profiles were $34276767050(n=22)$ and $34276763050(n=19)$. Each of these profiles was observed for three Cronobacter species. Profile 34276767050 was produced by strains of C. sakazakii $(n=16), C$. malonaticus $(n=4)$, and $C$. turicensis $(n=2)$. According to the updated ID32E database (version 4.0), these strains were all identified as C. sakazakii; however this result is a match for only $16 / 22$ strains (72.3 \%). Similarly, profile 34276763050 was observed for C. sakazakii $(n=16), C$. malonaticus $(n=2)$, and C. turicensis $(n=1)$. The species identification for this profile reported by the updated database was $C$. dublinensis. Though correct to the genus level, none of the strains producing this profile resulted in a match to the species level. It should also be noted that these three most common profiles differ from one another in only two tests, $\alpha$-glucosidase activity and trehalose acidification.

Both the API20E and ID32E biochemical kits include a test for inositol fermentation. Of the 61 API20E profiles, $62.3 \%$ were positive for inositol fermentation. A total of 159 strains were identified as being inositol positive with this test kit, including 112 strains of C. sakazakii. With the ID32E test kit $80.7 \%$ of the 155 unique profiles were positive for inositol fermentation. Of the 280 strains identified as being inositol positive with the ID32E test kit, 192 were C. sakazakii. As a major pathogenic species in the genus, the high number of $C$. sakazakii strains showing positive results for inositol fermentation is interesting due to the association of the trait with virulence [31]; however, this result may be due to over representation of clinical isolates of C. sakazakii in the culture collection and further investigation may be necessary for confirmation.

Two of the major limitations of the API20E and ID32E kits are that they rely on a limited number of tests (20 or 32, respectively) and are subjectively based on visual detection of a color change when reporting results. Thus, the interpretation of the colors by an individual researcher could affect the reported profile and subsequent species identification. The Vitek system attempts to avoid these problems using 64 biochemical tests and automated detection of the color changes for more consistent results. Using a subset of strains, the
Vitek system identified all 14 Cronobacter strains as members of the "Cronobacter sakazakii group"; Table 2. Although this term has no taxonomic standing and has not been defined, it was taken here as being equivalent to the Cronobacter genus. In contrast, the API20E misidentified 8/14 (57.1\%) of these strains to the genus level and the ID32E misidentified 13/14 (92.9\%) to the species level. Notably, though they were assigned to the 'C. sakazakii group' with the Vitek GN card, the type strains for C. condimenti $\left(\mathrm{LMG} 26250^{\mathrm{T}}\right)$, C. turicensis $\left(\mathrm{LMG} 23827^{\mathrm{T}}\right)$, C. universalis $\left(\mathrm{NCTC}^{2} 29^{\mathrm{T}}\right)$ and C. dublinensis $\left(\mathrm{LMG} 23823^{\mathrm{T}}\right.$ ) were misidentified with both the API20E and ID32E test kits; Table 2. The ID32E kit did identify these strains as members of the Cronobacter genus, but did not correctly identify these type strains to species level.

In addition, the Vitek GN card misidentified members of the Franconibacter genus as members of the " $C$. sakazakii group". The type strain of $F$. helveticus $\left(\right.$ LMG23732 ${ }^{\mathrm{T}}$ ) was also identified as a member of the Cronobacter genus by the other two test kits, while the type strain of F. pulveris (LMG24057 ${ }^{\mathrm{T}}$ ) was misidentified with the API20E test panel; Table 2. As mentioned previously, this not unexpected as current strains in the Franconibacter genus were briefly considered to be a part of the Cronobacter genus before Franconibacter was defined $[13,15]$. The false positive results generated by the Franconibacter species with the Vitek system are important for PIF manufacturers, as these species have not been linked to any cases of human illness but occur in similar ecosystems as Cronobacter species [15]. The international microbiological criteria applied to PIF require the absence of all Cronobacter species and this misidentification may cause manufacturers to incorrectly reject a batch of microbiologically safe infant formula.

Of the three biochemical methods evaluated in this study, the Vitek system performed better than the API20E or ID32E for identification of a subset of strains, including the type strains for all Cronobacter species and strains which had been previously misidentified. Though some false positive results were observed, this method was able to identify all tested Cronobacter species as members of the "Cronobacter sakazakii group;" however, relatively few strains were analysed with this method and analysis of a larger number of strains would be necessary to properly determine the accuracy of the Vitek system for identification of Cronobacter isolates. The FDA BAM method does suggest using either the Vitek 2 GN cards or the ID32E biochemical test panel for identification of suspect isolates, but there is a high cost associated with the initial purchase of the Vitek 2 instrument. Thus, while this method did seem to perform better in this small-scale analysis, the technology 
Table 2 Comparison of the API20E, ID32E, and Vitek GN methods for identification of Cronobacter and closely related species

\begin{tabular}{|c|c|c|c|c|c|c|}
\hline \multirow[b]{2}{*}{ Strain } & \multirow[b]{2}{*}{ Species } & \multirow{2}{*}{$\begin{array}{l}\text { Vitek GN } \\
\text { Identification }\end{array}$} & \multicolumn{2}{|l|}{ API20E } & \multicolumn{2}{|l|}{ ID32E } \\
\hline & & & Profile & $\begin{array}{l}\text { Species identification } \\
\text { (v5.0) }\end{array}$ & Profile & $\begin{array}{l}\text { Species identification } \\
\text { (V4.0) }\end{array}$ \\
\hline${\operatorname{LMG} 26250^{\top}}^{\top}$ & Cronobacter condimenti & Cronobacter sakazakii group & 3367373 & Cronobacter spp. & 34217360051 & Cronobacter muytjensii \\
\hline 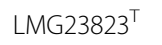 & Cronobacter. dublinensis & Cronobacter sakazakii group & 7347373 & Cronobacter spp. & 34256166211 & Cronobacter malonaticus \\
\hline 8 & Cronobacter malonaticus & Cronobacter sakazakii group & 3004153 & Escherichia vulneris & 34774563051 & Cronobacter malonaticus \\
\hline 16 & Cronobacter muytjensii & Cronobacter sakazakii group & 0004153 & Serratia plymuthica & 30676563051 & Cronobacter malonaticus \\
\hline 4 & Cronobacter sakazakii & Cronobacter sakazakii group & 3305173 & Pantoea spp. 3 & 34074743011 & Enterobacter cloacae \\
\hline 5 & Cronobacter sakazakii & Cronobacter sakazakii group & 3305173 & Pantoea spp. 4 & 14074743211 & Enterobacter amnigenus \\
\hline 12 & Cronobacter sakazakii & Cronobacter sakazakii group & 0005173 & Escherichia vulneris & 30674773050 & Cronobacter malonaticus \\
\hline 1436 & Cronobacter sakazakii & Cronobacter sakazakii group & 3305373 & Cronobacter spp. & 34276763011 & Cronobacter malonaticus \\
\hline 1437 & Cronobacter sakazakii & Cronobacter sakazakii group & 3305173 & Cronobacter spp. & 34276763010 & Cronobacter malonaticus \\
\hline 1438 & Cronobacter sakazakii & Cronobacter sakazakii group & 1304373 & Enterobacter gergoviae & 14234767010 & Cronobacter malonaticus \\
\hline 9 & Cronobacter turicensis & Cronobacter sakazakii group & 1006523 & Cronobacter spp. & 30074773050 & Enterobacter cloacae \\
\hline LMG $23827^{\top}$ & Cronobacter turicensis & Cronobacter sakazakii group & 7315373 & Enterobacter gergoviae & 34276767211 & Cronobacter malonaticus \\
\hline NCTC9529 & Cronobacter universalis & Cronobacter sakazakii group & 3205373 & Escherichia vulneris & 24276777051 & Cronobacter malonaticus \\
\hline 1435 & Cronobacter universalis & Cronobacter sakazakii group & 3304373 & Cronobacter spp. & 34356767010 & Cronobacter sakazakii \\
\hline 162 & Escherichia hermanii & Pantoea spp. & 1004153 & Cronobacter spp. & 34676767050 & Cronobacter sakazakii \\
\hline LMG23732 ${ }^{\top}$ & Franconibacter helveticus & Cronobacter sakazakii group & 1024153 & Cronobacter spp. & 30675567010 & Cronobacter sakazakii \\
\hline 1393 & Franconibacter pulveris & Cronobacter sakazakii group & 3004173 & Cronobacter spp. & 04275763310 & Enterobacter cloacae \\
\hline LMG24057 & Franconibacter pulveris & Cronobacter sakazakii group & 3004173 & Escherichia vulneris & 04275773310 & Enterobacter cloacae \\
\hline NCTC14934 ${ }^{\top}$ & Siccibacter colletis & Pantoea spp. & 3204153 & Escherichia vulneris & 04077563310 & Buttauxiella agrestis \\
\hline
\end{tabular}

may unfortunately not be accessible to all PIF manufacturers and food testing laboratories.

As reported here and by others, commercially available biochemical test panels are not sufficient for accurate identification of Cronobacter species and even show difficulties in identifying strains to the genus level [7, 20, 30, 32]. Thus, the use of an alternative method may be necessary for accurate species identification of suspect Cronobacter isolates from PIF. DNA sequence-based identification methods are more reliable, but also more expensive, labor-intensive and have a long turnaround time. As DNA sequencing becomes cheaper and easier, it may be possible to incorporate it into manufacturers' testing schemes, but this is not currently feasible. Similarly, while many other alternative methods are available for identification of Cronobacter spp., they may not be accessible to all laboratories. For example, real-time PCR methods and MALDI-TOF MS can be used to identify suspect isolates; however, these methods require expensive, specialized equipment and trained personnel that will prevent some laboratories from utilizing such techniques.

Several PCR probe-based identification methods have been proposed for the Cronobacter genus, which may provide an alternative to biochemical phenotyping or DNA sequence-based identification [22, 33-39]. These methods do not require any specialized equipment beyond a PCR thermocycler and gel electrophoresis capabilities, which would be expected in most, if not all, microbiology testing laboratories. Previous analysis comparing PCR probes with Cronobacter genome sequences showed the methods targeting the genes omp $A$ and $r p o B$ were most likely to result in accurate identification $[20,22,38,39]$. The $r p o B$ method requires a separate PCR primer pair and different amplification conditions for each of the seven Cronobacter species [38, 39]. In contrast, the ompA method only requires one primer pair and therefore is less laborious and has a shorter turnaround time [22]. Though the PCR method targeting ompA was developed to identify "E. sakazakii," strains previously known by this name were reclassified as members of the Cronobacter genus [5, 6]. The analysis of Jackson et al. showed that the method targeting $o m p A$ was able to identify the type strains of all seven Cronobacter species, while excluding closely related strains, particularly those belonging to the Franconibacter and Siccibacter genera [20, 22].

For a thorough examination of the ompA PCR method, in silico analysis of full genome sequences was used. This method enabled the analysis of a large cohort of internationally derived strains that are not centrally available. To determine if this method could be used for 
identification of Cronobacter from PIF, the genomes of 187 Cronobacter and 36 non-Cronobacter strains were examined for the presence of the primer sequences targeting ompA. Table 3 shows the number of mismatches with each primer and the expected amplicon size for each species. The ESSR-R primer showed a higher number of mismatches than the ESSR-F primer for non-Cronobacter species and all $F$. helveticus strains showed 7 mismatches with the ESSR-R primer. Four of these strains were used in the previously reported laboratory evaluation of this method and none produced amplicons [20]. It was, therefore, concluded that strains with 7 or more mismatches to the ESSR-R primer would not produce amplicons with this method.

Table 3 Predicted PCR amplification of ompA locus

\begin{tabular}{|c|c|c|c|c|}
\hline \multirow{3}{*}{ Species } & \multirow{3}{*}{$\mathrm{n}$} & \multicolumn{2}{|c|}{ \# mismatches } & \multirow{3}{*}{$\begin{array}{l}\text { Predicted amplicon } \\
\text { size }(b p)^{b}\end{array}$} \\
\hline & & $\overline{\text { ESSR-F }^{a}}$ & ESSR-R & \\
\hline & & (22 bp) & (19 bp) & \\
\hline Cronobacter sakazakii & 101 & 0 & $1-2$ & 468 \\
\hline Cronobacter malonaticus & 45 & 0 & 2 & 468 \\
\hline Cronobacter dublinensis & 14 & 0 & 2 & 469 \\
\hline Cronobacter turicensis & 10 & 0 & 2 & 468 \\
\hline Cronobacter muytjensi & 9 & 0 & 0 & 469 \\
\hline Cronobacter universalis & 7 & 0 & 2 & 468 \\
\hline Cronobacter condimenti & 1 & 0 & 2 & 469 \\
\hline Franconibacter pulveris & 7 & 1 & 8 & NA \\
\hline Franconibacter helveticus & 5 & 2 & 7 & NA \\
\hline Siccibacter turicensis & 4 & 4 & 10 & NA \\
\hline Enterobacter asburiae & 2 & $4-6$ & $8-11$ & NA \\
\hline Enterobacter hormaechei & 2 & $6-7$ & 8 & NA \\
\hline Enterobacter spp. & 2 & $5-6$ & 7 & NA \\
\hline Citrobacter amalonaticus & 1 & 7 & 9 & NA \\
\hline Citrobacter freundii & 1 & 6 & 12 & NA \\
\hline Citrobacter koseri & 1 & 7 & 13 & NA \\
\hline Citrobacter rodentium & 1 & 7 & 13 & NA \\
\hline Citrobacter youngae & 1 & 6 & 13 & NA \\
\hline Enterobacter aerogenes & 1 & 5 & 13 & NA \\
\hline Enterobacter cancerogenus & 1 & 5 & 7 & NA \\
\hline Enterobacter cloacae & 1 & 7 & 8 & NA \\
\hline Enterobacter ludwiggi & 1 & 5 & 7 & NA \\
\hline Enterobacter massilensis & 1 & 5 & 10 & NA \\
\hline Enterobacter mori & 1 & 5 & 7 & NA \\
\hline Enterobacter sacchari & 1 & 7 & 15 & NA \\
\hline Siccibacter colletis & 1 & 4 & 10 & NA \\
\hline Yersinia regensburgii & 1 & 5 & 7 & NA \\
\hline
\end{tabular}

aprimer sequences described previously [22]

${ }^{\mathrm{b}}$ Expected amplicon size: 469 bp [22]
Based on the in silico analysis of the ompA gene, none of the non-Cronobacter species examined would be expected to produce amplicons with this method. On the other hand, all Cronobacter spp. were predicted to produce amplicons of approximately the expected size. Mohan-Nair and Venkitanarayanan reported an expected size of $469 \mathrm{bp}$, while the in silico analysis predicted amplicons of 468-469 bp [22]. This in silico analysis indicates that this method should be sufficient to identify all Cronobacter species, while excluding members of closely related genera. Though further identification would be needed to identify the isolates to the species level, this would not be necessary for PIF manufacturers to ensure exclusion of all members of the Cronobacter genus from their products [1,27-29]. As this method is capable of identifying all Cronobacter spp., it could be used in place of the biochemical test panels for more accurate genus-level identification of suspect Cronobacter isolates from PIF.

\section{Conclusions}

Commercially available biochemical test panels, such as the API20E and ID32E, are not sufficient to identify Cronobacter isolates at the species level and reliance on these methods will result in false positive and false negative identifications. Only about $80 \%$ of Cronobacter strains were correctly identified to the genus level with current versions of the databases associated with either the AP20E or ID32E test kits. Identification to the species level with the ID32E kit resulted in a match for fewer than half of the strains. Though the Vitek GN cards identified all Cronobacter strains as members of the 'C. sakazakii group', members of the Franconibacter were also assigned to this group. In contrast, in silico analysis of the PCR probe-based method targeting ompA predicted this method could accurately and specifically identify members of the Cronobacter genus. While it cannot identify the individual species, this method would be sufficient for manufacturers to ensure the absence of all Cronobacter species in PIF as specified by international microbiological criteria. DNA sequence-based methods are more reliable for species identification, but are less practical for PIF manufacturers. Until DNA sequence-based identification is more feasible for routine microbiological testing laboratories, Cronobacter species can be identified using a combination of cultural, biochemical and/or molecular methods; however such methods are not applicable for microbial source tracking and epidemiological purposes. Nevertheless, correct genus level identification of suspect Cronobacter isolates will ensure that safe products reach consumers and will minimize productivity losses for PIF manufacturers. 


\section{Additional files}

Additional file 1: Table S1. Cronobacter spp. identifications using previous and current versions of the API20E database. (XLSX $12 \mathrm{~kb}$ )

Additional file 2: Table S2. Cronobacter spp. identifications using previous and current versions of the ID32E database. (XLSX $14 \mathrm{~kb}$ )

\section{Acknowledgements}

This publication made use of the Cronobacter Multi Locus Sequence Typing website developed by Keith Jolley and sited at the University of Oxford [17, 21]. The development of this site has been funded by the Wellcome Trust.

\section{Authors' contributions}

EEJ and SJF conceived the study. EEJ performed the analyses. Both authors prepared the manuscript and approved of the final version.

\section{Competing interests}

The authors declare that they have no competing interests.

Received: 19 April 2016 Accepted: 8 July 2016

Published online: 11 July 2016

\section{References}

1. Codex Alimentarius Commission. Code of hygienic practice for powdered formulae for infants and young children. 2008

2. Centers for Disease Control. CDC Update: Investigation of Cronobacter infections among infants in the United States. Available at: http://www.cdc. gov/cronobacter/investigation.html. Accessed 5 Apr 2016.

3. Geller, M. FDA says no need to recall Enfamil formula. Reuters 2011. Available at: http://www.reuters.com/article/us-meadjohnsonidUSTRE7BL17R20111231\#zbiwXoWXUhfzEyUv.97. Accessed 5 Apr 2016.

4. Minor T, Lasher A, Klontz K, Brown B, Nardinelli C, Zorn D. The per case and total annual costs of foodborne illness in the United States. Risk Anal. 2015; 35(6):1125-39.

5. Iversen C, Lehner A, Mullane N, Bidlas E, Cleenwerck I, Marugg J, Fanning S, Stephan $\mathrm{R}$, Joosten $\mathrm{H}$. The taxonomy of Enterobacter sakazakii: proposal of a new genus Cronobacter gen. nov. and descriptions of Cronobacter sakazakii comb. nov. Cronobacter sakazakii subsp. sakazakii, comb. nov., Cronobacter sakazakii subsp. malonaticus subsp. nov., Cronobacter turicensis sp. nov., Cronobacter muytjensii sp. nov., Cronobacter dublinensis sp. nov. and Cronobacter genomospecies 1. BMC Evol Biol. 2007;7:64.

6. Iversen C, Mullane N, McCardell B, Tall BD, Lehner A, Fanning S, Stephan R, Joosten $\mathrm{H}$. Cronobacter gen. nov., a new genus to accommodate the biogroups of Enterobacter sakazakii, and proposal of Cronobacter sakazakii gen. nov., comb. nov., Cronobacter malonaticus sp. nov., Cronobacter turicensis sp. nov., Cronobacter muytjensii sp. nov., Cronobacter dublinensis sp. nov. Cronobacter genomospecies 1, and of three subspecies, Cronobacter dublinensis subsp. dublinensis subsp. nov., Cronobacter dublinensis subsp. lausannensis subsp. nov. and Cronobacter dublinensis subsp. lactaridi subsp. nov. Int J Syst Evol Microbiol. 2008;58(Pt 6):1442-7.

7. Joseph S, Hariri S, Forsythe SJ. Lack of continuity between Cronobacter biotypes and species as determined using multilocus sequence typing. Mol Cell Probes. 2013;27(3-4):137-9.

8. Forsythe SJ, Dickins B, Jolley KA. Cronobacter, the emergent bacterial pathogen Enterobacter sakazakii comes of age; MLST and whole genome sequence analysis. BMC Genomics. 2014;15:1121.

9. Besse NG, Leclercq A, Maladen V, Tyburski C, Bertrand L. Evaluation of the International Organization for Standardization-International Dairy Federation (ISO-IDF) draft standard method for detection of Enterobacter sakazakii in powdered infant food formulas. J AOAC Int. 2006;89(5):1309-16.

10. International Organization for Standardization (ISO). Draft method: Microbiology of the food chain - Horizontal method for the detection of Cronobacter spp. Available at: http://www.iso.org/iso/home/store/catalogue_ ics/catalogue_detail_ics.htm?csnumber=64708. Accessed 7 June 2016.

11. Chen, Y., Lampel, K., Hammack, T. Bacteriological Analytical Manual, Chapter 29 Cronobacter. Available at: http://www.fda.gov/Food/FoodScienceResearch/ LaboratoryMethods/ucm289378.htm. Accessed 5 Apr 2016.

12. Iversen C, Lehner A, Mullane N, Marugg J, Fanning S, Stephan R, Joosten H. Identification of "Cronobacter" spp. (Enterobacter sakazakii). J Clin Microbiol. 2007;45(11):3814-6.
13. Brady C, Cleenwerck I, Venter S, Coutinho T, De Vos P. Taxonomic evaluation of the genus Enterobacter based on multilocus sequence analysis (MLSA): proposal to reclassify E. nimipressuralis and E. amnigenus into Lelliottia gen. nov. as Lelliottia nimipressuralis comb. nov. and Lelliottia amnigena comb. nov., respectively, E. gergoviae and E. pyrinus into Pluralibacter gen. nov. as Pluralibacter gergoviae comb. nov. and Pluralibacter pyrinus comb. nov., respectively, E. cowanii, E. radicincitans, E. oryzae and E. arachidis into Kosakonia gen. nov. as Kosakonia cowanii comb. nov., Kosakonia radicincitans comb. nov., Kosakonia oryzae comb. nov. and Kosakonia arachidis comb. nov., respectively, and E. turicensis, E. helveticus and E. pulveris into Cronobacter as Cronobacter zurichensis nom. nov., Cronobacter helveticus comb. nov. and Cronobacter pulveris comb. nov., respectively, and emended description of the genera Enterobacter and Cronobacter. Syst Appl Microbiol. 2013;36(5):309-19.

14. Jackson EE, Masood N, Ibrahim K, Urvoy N, Hariri S, Forsythe SJ. Description of Siccibacter colletis sp. nov., a novel species isolated from plant material, and emended description of Siccibacter turicensis. Int J Syst Evol Microbiol. 2015;65(Pt 4):1335-41.

15. Stephan R, Grim CJ, Gopinath GR, Mammel MK, Sathyamoorthy V, Trach LH, Chase HR, Fanning S, Tall BD. Re-examination of the taxonomic status of Enterobacter helveticus, Enterobacter pulveris and Enterobacter turicensis as members of the genus Cronobacter and their reclassification in the genera Franconibacter gen. nov. and Siccibacter gen. nov. as Franconibacter helveticus comb. nov., Franconibacter pulveris comb. nov. and Siccibacter turicensis comb. nov., respectively. Int J Syst Evol Microbiol. 2014;64(Pt 10):3402-10.

16. Baldwin A, Loughlin M, Caubilla-Barron J, Kucerova E, Manning G, Dowson C, Forsythe S. Multilocus sequence typing of Cronobacter sakazakii and Cronobacter malonaticus reveals stable clonal structures with clinical significance which do not correlate with biotypes. BMC Microbiol. 2009:9:223

17. Cronobacter PubMLST databases. http://www.pubmlst.org/cronobacter. Accessed 5 Apr 2016.

18. Cetinkaya E, Joseph S, Ayhan K, Forsythe SJ. Comparison of methods for the microbiological identification and profiling of Cronobacter species from ingredients used in the preparation of infant formula. Mol Cell Probes. 2013; 27:60-4.

19. Iversen C, Druggan P, Forsythe S. A selective differential medium for Enterobacter sakazakii, a preliminary study. Int J Food Microbiol. 2004;96(2):133-9.

20. Jackson EE, Sonbol H, Masood N, Forsythe SJ. Genotypic and phenotypic characteristics of Cronobacter species, with particular attention to the newly reclassified species Cronobacter helveticus, Cronobacter pulveris, and Cronobacter zurichensis. Food Microbiol. 2014;44:226-35.

21. Jolley KA, Maiden MC. BIGSdb: Scalable analysis of bacterial genome variation at the population level. BMC Bioinformatics. 2010;11(1):595.

22. Mohan Nair MK, Venkitanarayanan KS. Cloning and sequencing of the ompA gene of Enterobacter sakazakii and development of an ompA-targeted PCR for rapid detection of Enterobacter sakazakii in infant formula. Appl Environ Microbiol. 2006;72(4):2539-46.

23. Tamura K, Stecher G, Peterson D, Filipski A, Kumar S. MEGA6: Molecular Evolutionary Genetics Analysis version 6.0. Mol Biol Evol. 2013;30(12):2725-9.

24. Flores JP, Medrano SA, Sanchez JS, Fernandez-Escartin E. Two cases of hemorrhagic diarrhea caused by Cronobacter sakazakii in hospitalized nursing infants associated with the consumption of powdered infant formula. J Food Prot. 2011;74(12):2177-81.

25. Jackson EE, Flores JP, Fernandez-Escartin E, Forsythe SJ. Reevaluation of a suspected Cronobacter sakazakii outbreak in Mexico. J Food Prot. 2015;78(6): 1191-6.

26. Townsend SM, Hurrell E, Caubilla-Barron J, Loc-Carrillo C, Forsythe SJ. Characterization of an extended-spectrum beta-lactamase Enterobacter hormaechei nosocomial outbreak, and other Enterobacter hormaechei misidentified as Cronobacter (Enterobacter) sakazakii. Microbiology. 2008; 154(Pt 12):3659-67.

27. Food and Agricultural Organization-World Health Organization (FAO-WHO). Enterobacter sakazakii (Cronobacter spp.) in powdered formulae: Meeting report. Microbial Risk Assessment 2008, MRA Series 15.

28. Food and Agricultural Organization-World Health Organization (FAO-WHO). Enterobacter sakazakii and Salmonella in powdered infant formula. 2006, MRA Series 10.

29. Food and Agricultural Organization-World Health Organization (FAO-WHO). Enterobacter sakazakii and other organisms in powdered infant formula. 2004, MRA Series 6. 
30. Fanjat N, Leclercq A, Joosten H, Robichon D. Comparison of the phenotyping methods ID $32 E$ and VITEK 2 compact GN with 165 rRNA gene sequencing for the identification of Enterobacter sakazakii. J Clin Microbiol. 2007;45(6):2048-50.

31. Hamby SE, Joseph S, Forsythe SJ, Chuzhanova N. In silico identification of pathogenic strains of Cronobacter from biochemical data reveals association of inositol fermentation with pathogenicity. BMC Microbiol. 2011;11:204.

32. Jaradat ZW, Ababneh QO, Saadoun IM, Samara NA, Rashdan AM. Isolation of Cronobacter spp. (formerly Enterobacter sakazakii) from infant food, herbs and environmental samples and the subsequent identification and confirmation of the isolates using biochemical, chromogenic assays, PCR and 16S rRNA sequencing. BMC Microbiol. 2009;9:225.

33. Carter L, Lindsey LA, Grim CJ, Sathyamoorthy V, Jarvis KG, Gopinath G, Lee C, Sadowski JA, Trach L, Pava-Ripoll M, McCardell BA, Tall BD, Hu L. Multiplex PCR assay targeting a diguanylate cyclase-encoding gene, cgcA, to differentiate species within the genus Cronobacter. Appl Environ Microbiol. 2013;79(2):734-7.

34. Grim C, Kothary M, Gopinath G, Jarvis K, Beaubrun JJ, McClelland M, Tall B, Franco A. Identification and characterization of Cronobacter iron acquisition systems. Appl Environ Microbiol. 2012;78(17):6035-50.

35. Hassan AA, Akineden O, Kress C, Estuningsih S, Schneider E, Usleber E. Characterization of the gene encoding the 16S rRNA of Enterobacter sakazakii and development of a species-specific PCR method. Int J Food Microbiol. 2007:116(2):214-20.

36. Kothary MH, McCardell BA, Frazar CD, Deer D, Tall BD. Characterization of the zinc-containing metalloprotease encoded by zpx and development of a species-specific detection method for Enterobacter sakazakii. Appl Environ Microbiol. 2007:73(13):4142-51

37. Lehner A, Tasara T, Stephan R. 16S rRNA gene based analysis of Enterobacter sakazakii strains from different sources and development of a PCR assay for identification. BMC Microbiol. 2004;4:43.

38. Lehner A, Fricker-Feer C, Stephan R. Identification of the recently described Cronobacter condimenti by an rpoB-gene-based PCR system. J Med Microbiol. 2012;61(Pt 7):1034-5.

39. Stoop B, Lehner A, Iversen C, Fanning S, Stephan R. Development and evaluation of rpoB based PCR systems to differentiate the six proposed species within the genus Cronobacter. Int J Food Microbiol. 2009;136(2):165-8.

\section{Submit your next manuscript to BioMed Central and we will help you at every step:}

- We accept pre-submission inquiries

- Our selector tool helps you to find the most relevant journal

- We provide round the clock customer support

- Convenient online submission

- Thorough peer review

- Inclusion in PubMed and all major indexing services

- Maximum visibility for your research

Submit your manuscript at www biomedcentral.com/submit

) Biomed Central 